\title{
Influence of Geometries on the Assembly of Snowman-Shaped Janus Nanoparticles
}

\author{
Chengjun Kang $\left.{ }^{(}\right)$and Andrei Honciuc*(i) \\ Institute of Chemistry and Biotechnology, Zurich University of Applied Sciences, Einsiedlerstrasse 31, 8820 Waedenswil, Switzerland
}

Supporting Information

\begin{abstract}
The self-assembly of micro/nanoparticles into suprastructures is a promising way to develop reconfigurable materials and to gain insights into the fundamental question of how matter organizes itself. The geometry of particles, especially those deviating from perfectly spherical shapes, is of significant importance in colloidal assembly because it influences the particle "recognition", determines the particle packing, and ultimately dictates the formation of assembled suprastructures. In order to organize particles into desired structures, it is of vital importance to understand the relationship between the shape of the colloidal building blocks and the assembled suprastructures. This fundamental issue is an enduring topic in the assembly of molecular surfactants, but it remained elusive in colloidal assembly. To address this issue, we use snowman-shaped Janus nanoparticles (JNPs) as a model to systematically study the effect of colloidal geometries on their assembled suprastructures. Ten types of JNPs with identical chemical compositions but with different geometries were synthesized. Specifically, the synthesized JNPs differ in their lobe size

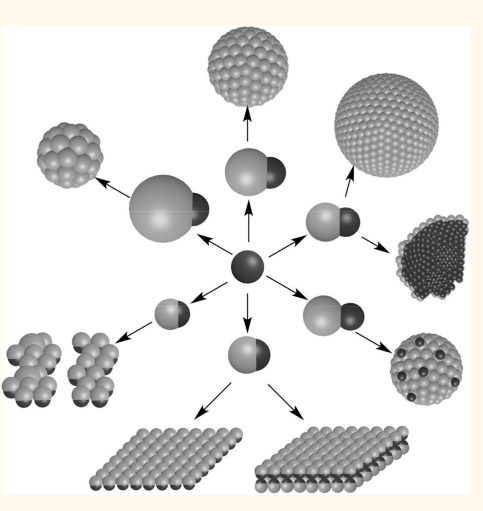
ratios, phase separation degrees, and overall sizes. We show that by altering these parameters, both finite suprastructures, such as capsules with different curvatures, and nonfinite suprastructures, including free-standing single-layered or doublelayered JNPs sheets, can be obtained via self-assembly. All these different types of suprastructures are constituted by highly oriented and hexagonally packed JNPs. These findings demonstrate the significance of geometries in colloidal assembly, such that slightly changing the building block geometries could result in a large variety of very different assembled structures, without altering the chemistry of the particles.
\end{abstract}

KEYWORDS: colloidal assembly, Janus nanoparticles, asymmetric particles, particle suprastructures, vesicles, coassembly

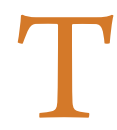
he interest in manipulating the self-assembly of micro/ nanoparticles into ordered suprastructures has long been motivated by their wide applications in photonics, ${ }^{1,2}$ electronics, ${ }^{3,4}$ and catalysis. ${ }^{5}$ Shape plays a vital role in colloidal self-assembly, as it can control particle interaction, "recognition", 6 packing, and thus the assembled structures. ${ }^{7-10}$ Therefore, as compared to spherical building blocks, anisotropic colloidal particles may yield a significantly larger variety of self-assembled suprastructures. ${ }^{11}$ Nature takes advantage of anisotropy in a variety of proteins, whereas the resulting self-assembly structures and their functional roles depend decisively on the anisotropy of the building blocks. ${ }^{12}$ As for Janus ellipsoid assembly, Monte Carlo simulations have shown that the size and structure of assembled aggregates are largely dictated by the ellipsoid aspect ratio, ${ }^{13}$ and prolate spheroids could form various ordered cluster structures. ${ }^{14}$

The recent progress in particle synthesis has led to a revolution in synthesizing nano- and microsized colloidal particles with anisotropic geometries. Colloidal particles with a variety of morphologies, such as triangles cubes, ${ }^{15}$ tetrahedra, $^{16}$ stars, $^{17}$ and crescent moon shapes, ${ }^{18,19}$ among others, have been synthesized. These particles could be the "atoms" and "molecules" of future materials only if they could be assembled into well-ordered and useful structures. Anisotropic colloidal particles show interesting collective behavior, such as self-assembly into complex structures that show unusual properties. ${ }^{20}$ By exploring these collective phenomena, we could gain insights into general questions, such as how matter organizes itself, a prevalent topic in condensed-matter physics, materials science, and biology. ${ }^{20}$ Unfortunately, in contrast to a large variety of synthesized colloidal building blocks, only a small portion has been successfully assembled into regular structures, such as trimers, ${ }^{21}$ spherical micelles, ${ }^{22}$ capsules, ${ }^{23}$ and colloidal crystals. ${ }^{24}$ Because the interactions between colloidal particles are often shortranged, the self-assembly or the packing of the particles is highly affected by their geometrical or topological constraints. $^{20,25}$ Therefore, it is of great interest to establish the correlations between the geometry of individual colloidal building blocks and the resulting assembled structures. In order to attack this issue, a fully controlled synthesis of colloidal particles with identical chemical compositions but having

Received: February 5, 2018

Accepted: March 1, 2018 

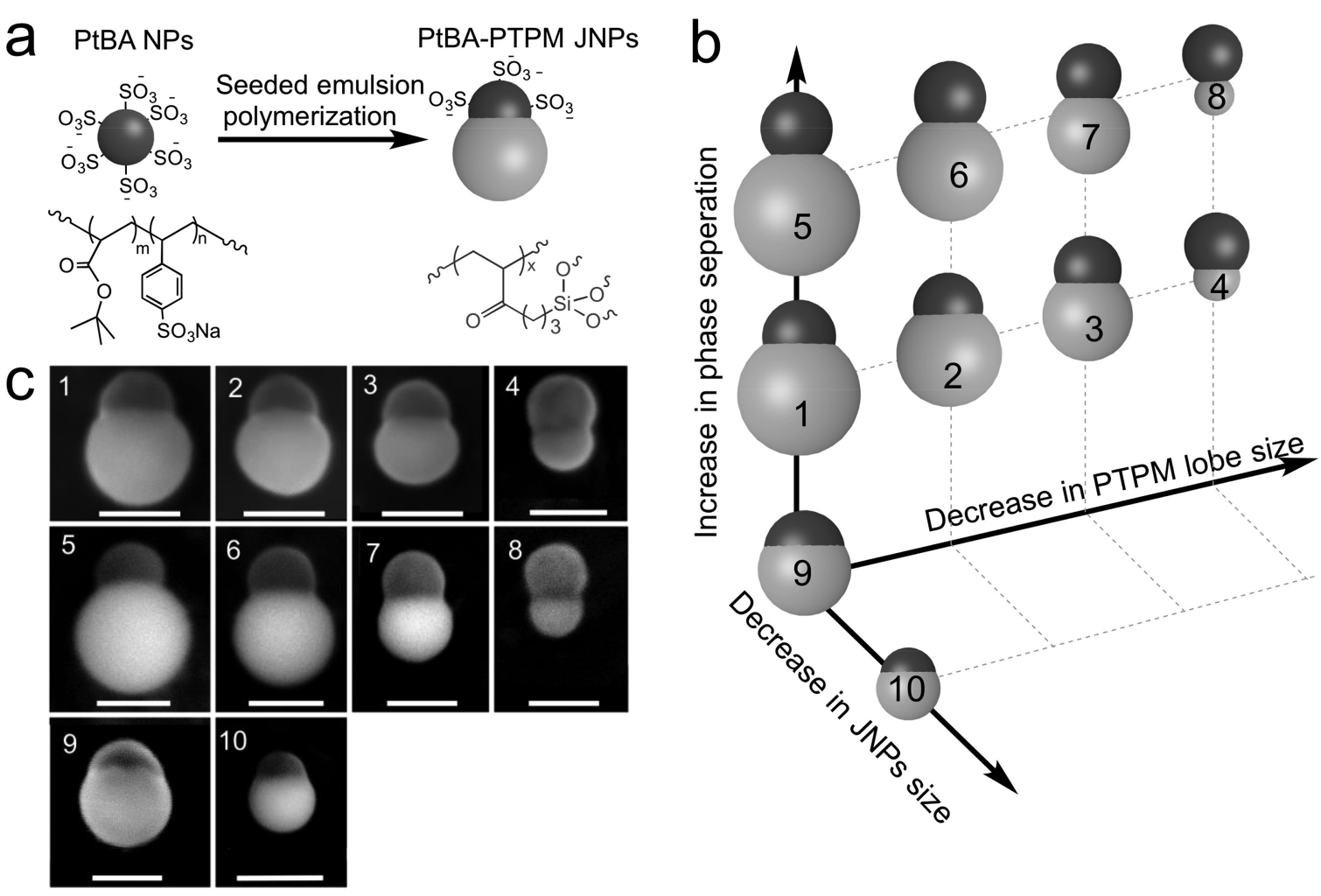

Figure 1. Synthesis of PtBA-PTPM JNPs with different geometries. (a) Synthesis of JNPs by seeded emulsion polymerization. (b) Summary of JNPs used in the present study. These JNPs vary in the PTPM lobe size, phase separation degree, and overall dimension. (c) SEM images of JNPs in (b). Scale bars are $150 \mathrm{~nm}$.

different geometries is required. Furthermore, reliable ways are demanded to assemble these particles of different geometries into ordered structures, which remains a challenging task, and, to the best of our knowledge, no equivalent work has been reported.

In the present study, we use snowman-shaped Janus nanoparticles (JNPs) as a model system to present a systematic study on the influence of geometries on colloidal self-assembly in a monophasic solvent. The reasons that we choose JNPs as a model system are as follows: (i) the structure of snowmanshaped JNPs is relatively simple, such that all the structural parameters can be fully controlled. Snowman-shaped JNPs are a unique class of anisotropic building blocks consisting of two different lobes, ${ }^{26,27}$ and both of them can be independently tuned in their dimensions and chemical compositions. These characteristics give snowman-shaped JNPs broken symmetry and molecular-like directionality via chemical anisotropy on two sides. (ii) Snowman-shaped JNPs can be conveniently synthesized in gram scale with high uniformity. Narrow polydispersed snowman-shaped JNPs can be conveniently synthesized by a seeded emulsion polymerization method in gram scale amount under surfactant-free conditions, ${ }^{28,29}$ ensuring sufficient samples for studying their assembly behaviors. (iii) Although structurally simple, snowman-shaped JNPs have a large number of self-assembly possibilities. A considerable amount of numerical simulations have highlighted a great assembly scenario, ${ }^{14,30-32}$ where JNPs can be selfassembled into suprastructures analogous to amphiphilic molecules or macromolecules, such as micelles, vesicles, or lamellar phases. Here we investigate the assembly of 10 types of JNPs, which are different in directionality, aspect ratio, and overall dimensions, in order to establish the correlations between their geometries and the types of self-assembled suprastructures formed.

\section{RESULTS AND DISCUSSION}

Synthesis of JNPs with Different Geometries. In the present study, we used JNPs poly(tert-butyl acrylate)-poly(3(triethoxysilyl)propyl methacrylate) (PtBA-PTPM) as model particles to study their self-assembly. Ten types of PtBA-PTPM JNPs were synthesized, they are geometrically different in three aspects, namely, the size of the PTPM lobe (controlling the directionality of JNPs), the PTPM lobe phase separation degree (controlling the aspect ratio of JNPs), and their overall dimensions (controlling the balance between the particle mobility and their interparticle attraction strength) (Figure 1).

Seeded emulsion polymerization is one of the most proficient methods for the synthesis of anisotropic Janus particles in gram-scale amount. The synthesized anisotropic colloids can be further transformed in other types of particles. For example Chen et al. ${ }^{33}$ used this method to synthesize sub-micrometer particles with a unique lens shape. In the present study, all the JNPs were synthesized by seeded-emulsion polymerization ${ }^{34,35}$ (Figure 1a), which allowed us to tune the JNPs' geometries in three different ways. First, the overall size of JNPs can be controlled by adjusting the size of the seed particles. In the first synthetic step, PtBA seed particles were synthesized by using the hydrophobic tert-butyl methacrylate (tBA) as the monomer, divinylbenzene (DVB) as the cross-linker, and sodium 4vinylbenzensulfonate (NaVBS) as the surfactant monomer. NaVBS was used because it can stabilize the seed NPs via electrostatic forces during the polymerization process. More importantly, by tuning the feeding ratios between the NaVBS and the $\mathrm{tBA}$, the size of the seed particles can be conveniently 

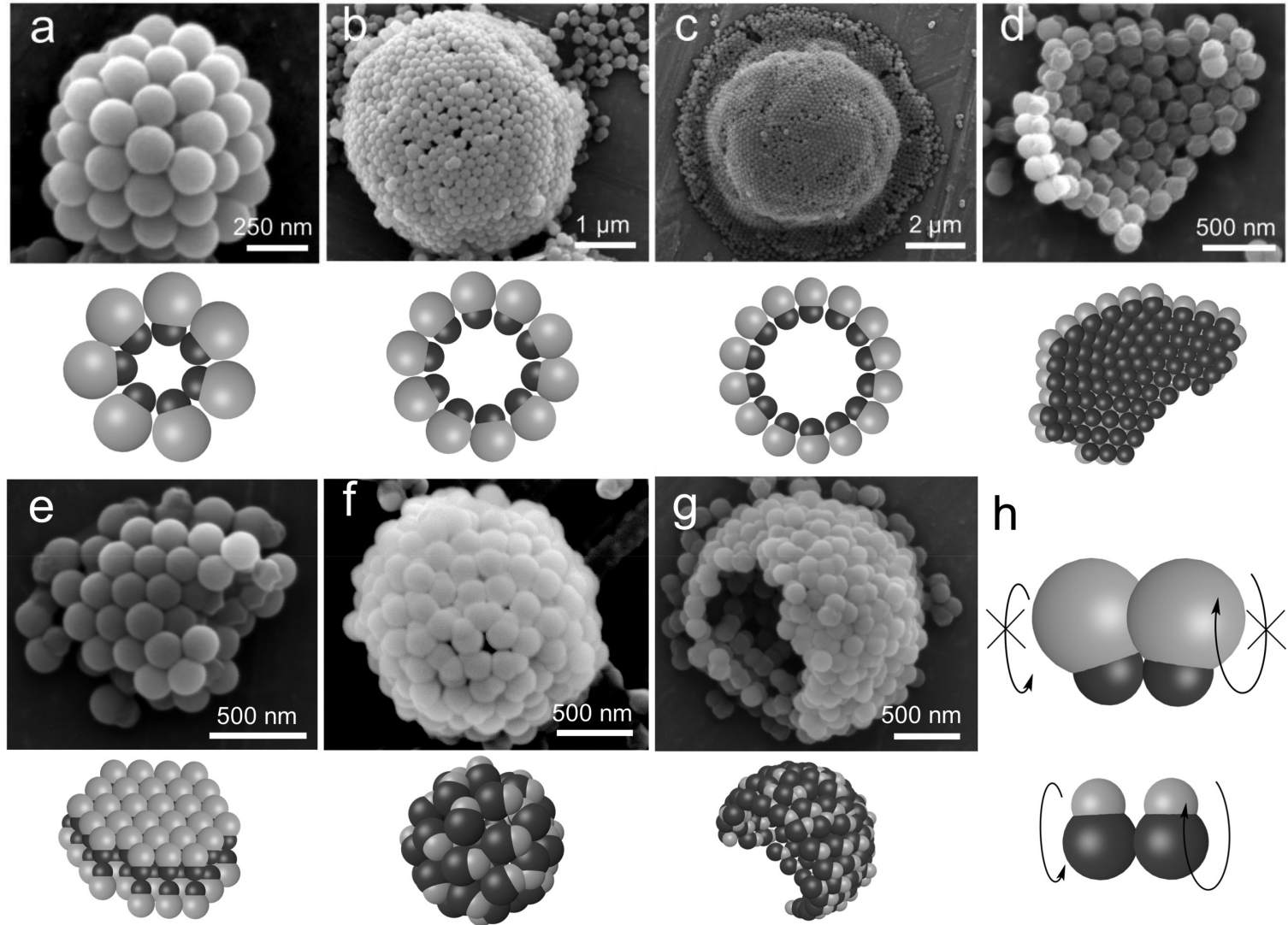

Figure 2. Self-assembled supracolloidal structures obtained from the PtBA-PTPM JNPs with different PTPM lobe sizes. (a-c) Capsules formed by JNP-1-JNP-3. (d, e) Monolayer (d) and double-layer (e) JNP sheets formed by JNP-3. (f, g) Capsule (f) and broken capsule (g) assembled by JNP-4. (h) JNPs with larger PTPM lobes (top) enjoy less rotational freedom as compared to the JNPs with smaller PTPM lobes (bottom).

adjusted. Here, seed particles with diameters in the dried state of $139 \pm 6 \mathrm{~nm}$ (JNPs 1-9) and $83 \pm 4 \mathrm{~nm}$ (JNP-10) were synthesized. Second, the size of the PTPM lobe can be tuned. Based on the seed NPs synthesized in the first step, JNPs were synthesized by seeded emulsion polymerization. The phase separation of 3-(triethoxysilyl)propyl methacrylate (TPM) from the PtBA seed NPs leads to the formation of PtBAPTPM JNPs. As for the same seed particles, JNPs with different PTPM lobe sizes can be conveniently obtained by varying the feed ratios between the seed NPs and TPM monomer. Lower TPM feeding ratios resulted in smaller PTPM lobes (from JNP1 to JNP-4). Third, JNPs with different PTPM phase separation degrees can be obtained. It is well known that as for the synthesis of JNPs by seeded emulsion polymerizations, the phase separation of the second polymer is highly sensitive to the surface chemistry of the seed particles. ${ }^{29}$ In the present study, the phase separation of the PTPM from PtBA seed particles can be conveniently controlled by tuning seed NP surface properties via polymerization time. In our experiments, we noticed that the size of the seed particles fully grew in $2 \mathrm{~h}$ and remained the same for the next $22 \mathrm{~h}$ of polymerization. However, as compared to the seed particles that polymerized for $2 \mathrm{~h}$ (JNP-9), $8 \mathrm{~h}$ (JNPs-1-4), and $24 \mathrm{~h}$ (JNPs-5-8), polymerized seed particles have higher phase separation degrees. This phenomenon could possibly be explained by the different polymerization speed between the NaVBS monomer and the tBA monomer. Because of the resonance effect, the radicals formed by NaVBS monomers are more stable than that of the tBA monomer; therefore, the NaVBS monomers polymerized relatively slower and the concentration of the poly(NaVBS) will gradually increase in the seed NPs with the polymerization time. Subsequently, within a certain time range, a longer polymerization time will make the seed NP surface relatively more hydrophilic and thus have a higher PTPM phase separation degree in the JNP formation process. Unfortunately, because the NaVBS concentration is so low, it is very challenging to directly probe any small variations of $-\mathrm{SO}_{3}{ }^{-}$amount on the seed JNP surface. In summary, by controlling the polymerization conditions, we could control the geometries of snowman-shaped JNPs. Specifically, JNP-1 to JNP-4 and JNP-5 to JNP-8 are JNPs with gradually decreasing PTPM lobe size. JNP-1, JNP-5, JNP-6, and JNP-2 are different in the phase separation degree of the PTPM lobe. The JNPs-9 and -10 have similar shape; however, they are different in their overall sizes (Figure $1 \mathrm{~b}$ ).

Influence of the PTPM Lobe Size on JNP SelfAssembly. The first geometric parameter is the relative lobe size. In order to form self-assembly structures, the hydrophobic attractions between the PtBA lobes are employed to bond individual JNPs together, while the PTPM lobe provides directionality to JNPs. Henceforth, we refer to the PtBA lobe as the "attraction lobe" and the PTPM lobe as the "nonattraction" lobe. In this section, we keep the PtBA lobe size constant and selectively change the size of the PTPM lobe. Figure 2 highlights different self-assembly structures that resulted from JNP-1 to JNP-4. The following observations can be made from Figure 2: (i) JNP-1, JNP-2, and JNP-3 can be assembled into spherical suprastructures, namely, spherical 


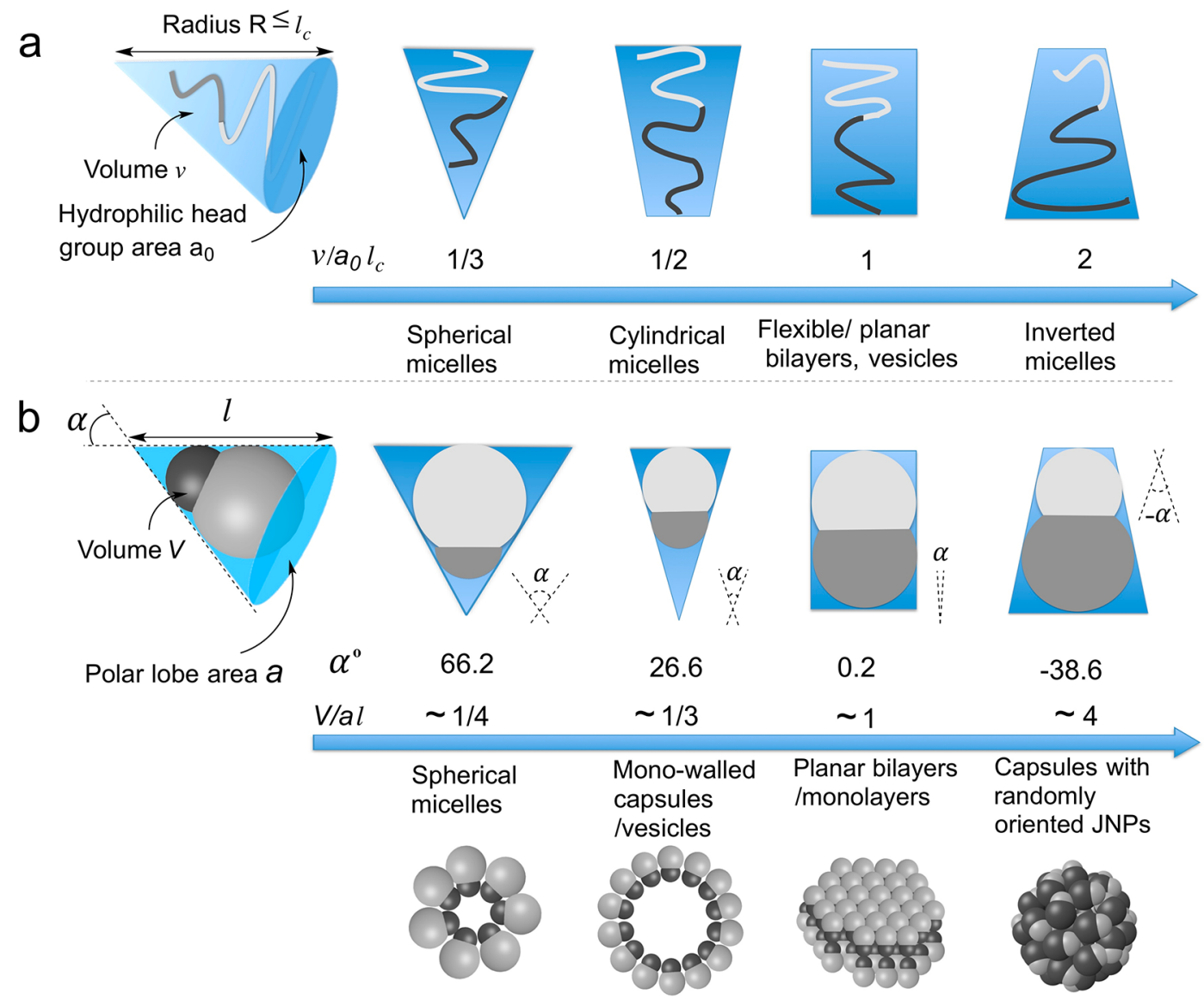

Figure 3. Comparison between surfactants and JNPs: (a) the critical packing parameter $\mathrm{Cpp}=\frac{v}{l_{c} a_{0}}$ with the idealized surfactant shapes for different self-assembled suprastructures. (b) Representation of the Janus critical packing parameters JCpp $=\frac{V}{l a}$. The angle $\alpha$ is measured from the actual dimensions of the particles. Note that $l$ is the height of the circumscribing structure.

micelles and capsules; capsules are void inside (Figure S1) and are constructed by hexagonally packed and highly oriented JNPs with the PTPM lobe pointing toward the outer shell. These suprastructures are big enough to be observed with the optical microscope, but individual JNPs cannot be resolved. By using fluorescent dye in the aqueous phase, we observed that water is present inside of the capsule because the fluorescent dye is uniformly distributed in- and outside of the capsules (Figure S2), which dismisses the hypothesis that air bubbles were introduced and act as the templates for suprastructures. SEM images with a larger field of view are shown in Figure S3. Decrease of the PTPM lobe size leads to larger suprastructures with lower curvature (Figure $2 \mathrm{a}-\mathrm{c}$ ). For example, as the PTPM lobe size decreases from $209 \pm 11 \mathrm{~nm}$ (JNP-1) to $162 \pm 6 \mathrm{~nm}$ (JNP-3), the size of the assembled capsules increases from 1.3 $\pm 0.5 \mu \mathrm{m}$ to $6.4 \pm 1.1 \mu \mathrm{m}$. (ii) Reduction of the PTPM lobe size to a certain extent results in the formation of different supracolloidal structures. For instance, as compared to the capsules formed by JNP-1 and JNP-2, JNP-3 can be assembled into two additional single- (Figure 2d) and double-layered (Figure 2e) free-standing JNP sheets. (iii) A dramatic change occurs when the PTPM lobe size decreased to such an extent that it becomes smaller than the PtBA lobe (JNP-4). In this case, even though capsules can be formed, the closely packed JNPs have their long axis positioned horizontally in the plane of the wall but without clear orientation (Figure $2 \mathrm{f}, \mathrm{g}$ ).
It is thus evident that the size ratio between the PtBA attraction lobe and the PTPM nonattraction lobe determines the curvatures of the self-assembled structures. Here, we introduce the concept of JNP "directionality" with the angle $\alpha$ (Figure 3). JNPs with larger absolute $\alpha$ values correspond to higher directionality and may self-assemble into structures with larger curvature. Therefore, we refer to the angle $\alpha$ as the curvature-determining parameter. When the PtBA lobe is smaller than the PTPM lobe, $\alpha>0$; when the PtBA and the PTPM lobes have exactly the same size, $\alpha=0$; when the PtBA lobe is bigger than the PTPM lobe, $\alpha<0$, denoting the JNPs have reverse directionality. From Figure 2a (JNP-1, $\alpha=66.2^{\circ}$ ) to Figure $2 \mathrm{c}\left(\mathrm{JNP}-3, \alpha=12.6^{\circ}\right)$ we can see that as the $\alpha$ decreases, the assembled structures transformed from micelles to capsules, which is accompanied by decreasing of their curvature and by increasing in dimension. The walls of these capsules are single layered and consist of highly oriented JNPs. Because of the geometric restriction, the capsule walls can only bend from the bigger PTPM lobe toward the smaller PtBA lobe. The formation of double-layered structures, as seen in vesicles formed by small molecules and block copolymers, this is also possible for the JNPs which have two lobes with similar sizes. Therefore, it is not surprising that when the $\alpha$ is low enough (JNP-3), we can see curved free-standing monolayers formed by highly oriented JNPs (Figure 2d). Interestingly, we also observed double-layered sheets (Figure 2e), in which the hydrophobic PtBA lobe is oriented inward while the relatively 

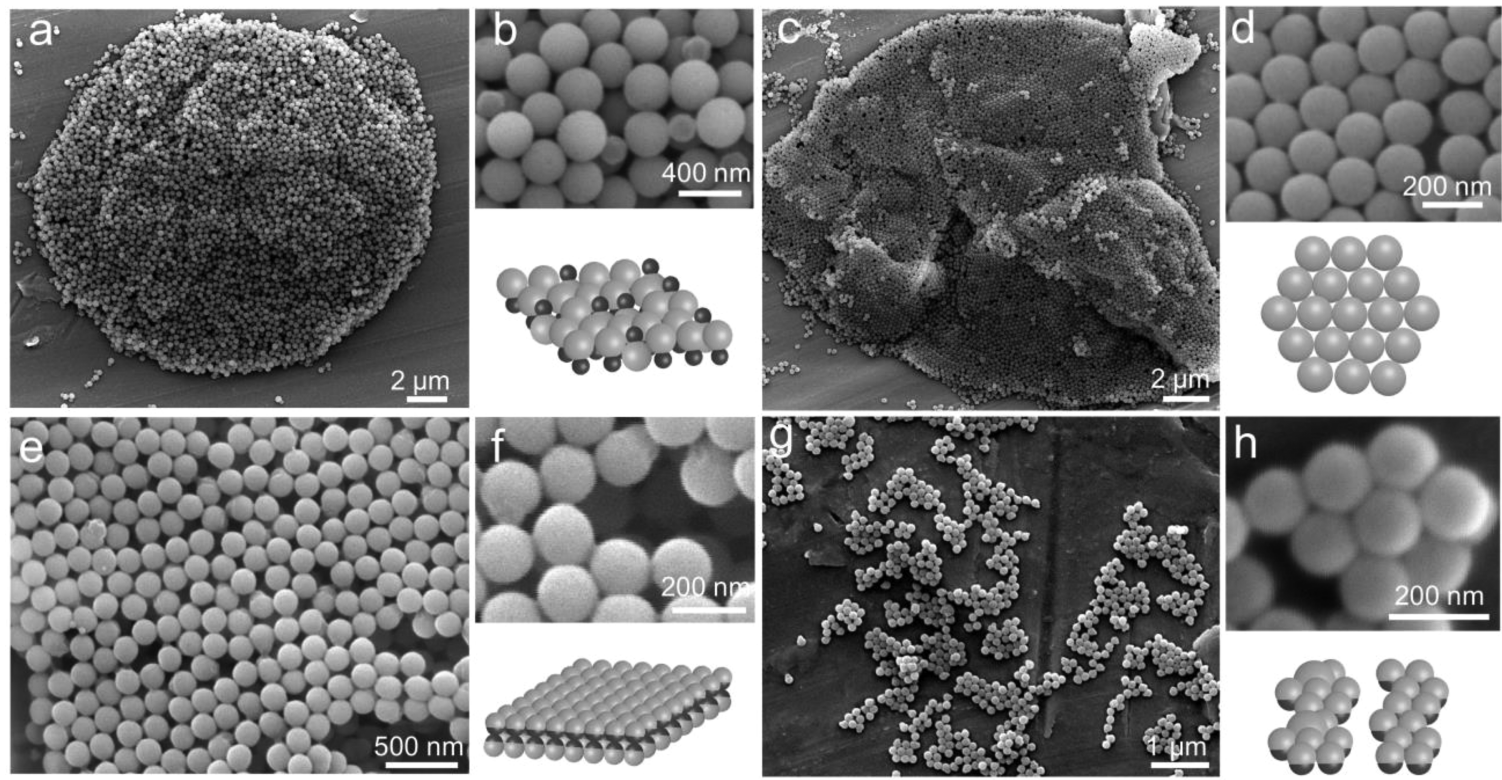

Figure 4. SEM images of suprastructures self-assembled by JNPs with different phase separation degrees. (a) Collapsed capsule assembled by JNP-5. (b) Partial magnification of (a). (c) Large-area JNP sheet self-assembled by JNP-9. (d) Partial magnification of (c). (e) Double-layerd JNP structure assembled by JNP-9. (f) Partial magnification of (e). (g) Island-like structures assembled by JNP-10. (h) Partial magnification of $(\mathrm{g})$.

hydrophilic PTPM lobe is uniformly oriented toward the aqueous solution. The freestanding double-layer JNP sheets are equivalent to the lipid bilayers, which are energetically preferred over the single-layered supracolloidal structures.

On further decrease in the PTPM lobe size $(121 \pm 8 \mathrm{~nm})$ until it becomes smaller than the PtBA lobe $(139 \pm 6 \mathrm{~nm})$ (JNP-4), $\alpha$ becomes negative. Because of the geometric restriction, it is reasonable to expect that the curvature of the suprastructure walls would bend from the bigger PtBA lobe toward the smaller PTPM lobe, resulting in curvature reversed capsules or micelles with the hydrophobic PtBA lobes pointing to the aqueous solution and the PTPM lobes pointing inward. However, we did not observe such reversed structures; what we observed mostly are the capsules consisting of randomly oriented JNP-4, even though the JNPs-4 have their long axis positioned horizontally in the plane of the capsule walls (Figure $2 f$ ). Broken capsules are occasionally encountered showing the void inside of these special capsules (Figure $2 \mathrm{~g}$ ). The formation of these unique capsules can be explained by the fact that the presence of the nonattractive PTPM lobe would both geometrically restrict the hydrophobic attraction and arrangement possibilities between the PtBA lobes and decrease the JNPs' rotational freedom during the self-assembly process (Figure 2h). The bigger the PTPM lobe is, the less freedom, especially rotational freedom, the JNPs enjoys with less possible ways for the PtBA lobes to bond together; thus these JNPs are more likely to form ordered suprastructures. Oppositely, when the PtBA lobe is larger than the PTPM lobe, the possible attraction points and rotation freedom between the PtBA lobe are largely increased (Figure $2 \mathrm{~h}$ ). The increased degree of freedom does result in more disordered and less well-defined suprastructures, as many more packing ways for the JNPs to fit into the supracolloidal structures become possible.

The correlations between the geometries of the surfactants and their ultimate assembled structures, such as micelles, planar bilayers, vesicles, etc., have been well-studied. ${ }^{36}$ In that case, a critical packing parameter $(\mathrm{Cpp})$ is defined.

$$
\mathrm{Cpp}=\frac{v}{l_{\mathrm{c}} a_{0}}
$$

where $v$ is the surfactant chain volume, $l_{c}$ is the critical surfactant chain length, and $a_{0}$ is the optimum headgroup area. Surfactants with smaller Cpp values have more cone-shape geometries (Figure 3). Because some of these parameters, such as its optimum headgroup area, are determined from the selfassembled structures, it appears that the idealization of the surfactant shape is rationalized post hoc. In order to better understand the effect of the JNPs' geometries on their assembled structures, we propose, in addition to the directional angle $\alpha$, the Janus critical packing parameter (JCpp). The JCpp was calculated from the measurements of the JNPs' structural parameters:

$$
\mathrm{JCpp}=\frac{V}{l a}
$$

where $V$ is the JNP's volume of geometrical shape-cone, cylinder, and truncated cone-defined by the tangent planes crossing at the side of the two Janus lobes (Figure 3 ). $l$ is the height of the corresponding cone or circular truncated cone or the height of the cylinder. $a$ is the bottom area of the corresponding cone, cylinder, or circular truncated cone. Because the PtBA lobe can be swollen in the aqueous solvent containing $10 \%(\mathrm{v} / \mathrm{v})$ ethanol, it should be noted that the above parameters were calculated directly from the swelled PtBA seed NPs in solvent $(153 \pm 6 \mathrm{~nm})$, as determined from the colloidal crystal (Figure S4). When the JCpp is around 1/4, spherical micelles were obtained, which is similar to the surfactant (Figure 3). However, as the JCpp increased to $1 / 3$, we did not obtain cylindrical micelles, but instead the monowalled capsules/vesicles. For JCpp $\approx 1$, mono- or bilayer JNP sheets were observed, in contrast to the bilayered suprastructures in all cases for surfactants. Finally, when the JCpp > 1, we observed capsules containing randomly oriented JNPs. 


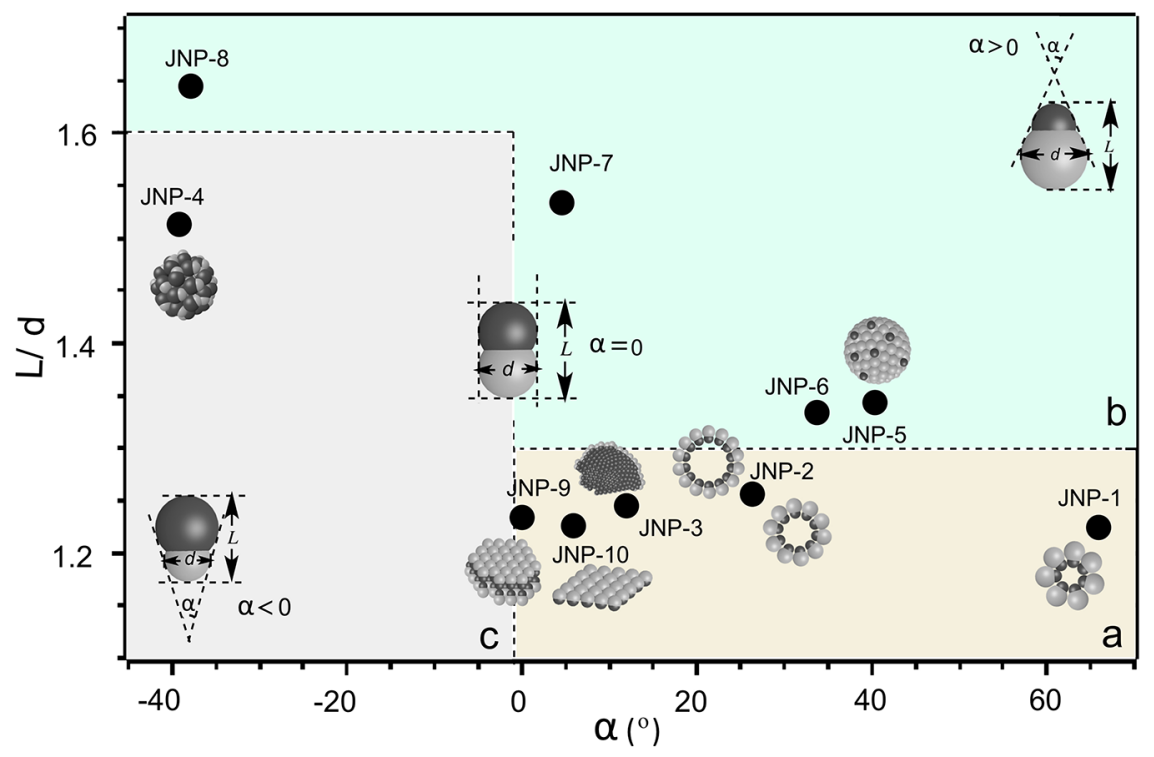

Figure 5. Phase diagram of suprastructures formed by JNPs with $\alpha$ (curvature determinant) as a function of $L / d$ (phase separation degree of the Janus lobes). (a) Domain containing JNPs with $L / d<1.3$ and $\alpha$ varying from $0^{\circ}$ to $70^{\circ}$. Various suprastructures composed by hexagonally packed and highly oriented JNPs can be obtained. (b) Domain containing JNPs with $L / d>1.3$ and $\alpha$ varying from $-40^{\circ}$ to $70^{\circ}$. No suprastructures consisting of well-oriented JNPs can be assembled in this area. (c) Domain containing JNPs with $\alpha<0$ when the PTPM lobe is smaller than the PtBA lobe.

Influence of the Aspect Ratio on JNP Self-Assembly. In order to study the influence of the aspect ratio on the JNPs' self-assembly properties, we compared those particles that share similar values of $\alpha$, but are different in their aspect ratios. By tuning the PTPM lobe phase separation degrees, we could change the JNPs' aspect ratio $\gamma$, which is defined by the ratio between the length of JNPs $(L)$ and the diameter of the PTPM lobe $(d), \gamma=L / d$. The assembled suprastructures of JNPs with different $\gamma$ values are shown in Figure 4. For instance, the JNP-2 and the JNP-6 have similar $\alpha$ values of $26.6^{\circ}$ and $33.7^{\circ}$, respectively; however, they are different in aspect ratio, which gives JNPs very different assembly properties (Figure 5). As we showed above, the JNP-2 can assemble into capsules composed by hexagonally packed and highly oriented JNPs. However, as the phase separation degree increases from JNP-2 $(\gamma=1.25)$ to JNP-6 $(\gamma=1.33)$, no ordered structures can be obtained under any conditions. This phenomenon could be explained as follows: higher phase separation degree implies larger PtBA surface area exposed between contacting JNPs and more bonding possibilities as well as packing ways of JNPs can be expected during the assembly process; subsequently, there is a lower likelihood of the formation of regular structures. It is also reasonable to expect that the JNPs with higher $\gamma$ values will have longer relaxation times for JNP rotation, so as to adjust their conformations to reach a uniform orientation. JNPs with longer relaxation times indicate that they are more likely to be frozen into aggregates without fully adjusting their orientation. This hypothesis is experimentally proved by the fact that no self-assembled suprastructure composed of highly oriented JNPs can be obtained (Figure 5) when both $\alpha$ (directionality) and $\gamma$ (phase separation) decrease, as in JNP-7 and -8. On the contrary, if the PTPM lobe size increases so as to enhance the JNPs' directionality (JNP-5), suprastructures composed by a certain degree of uniformly oriented JNPs can be obtained. From Figure 4a we can see that capsules with hexagonally packed JNP-5 can be formed. It is interesting to see that because of the high aspect ratio, JNP-5 did not orient completely uniformly, with some JNPs oriented in the opposite direction, Figure $4 \mathrm{~b}$. These reversely oriented JNPs act as defects in the capsules and make the capsule structures less compact and more prone to be broken by the capillary force during the drying process of the sample preparation for SEM (scanning electron microscope) measurements, which is the reason that almost all the observed capsules formed by JNP-5 are collapsed (Figure 4a).

On one hand, as compared to JNP-7, JNP-9 has a much lower aspect ratio $(\gamma=1.23)$. On the other hand, as compared to JNP-1, JNP-9 has reduced directionality $\left(\alpha=0.2^{\circ}\right)$. From these geometric characteristics and based on our above results, one could predict JNP-9's assembly behavior as follows: first, because JNP-9 may easily adjust its orientation during the assembly process, suprastructures containing highly oriented JNP-9 can be expected. Second, because of the reduced curvature, JNP-9 is prone to assemble into planar structures. Indeed, JNP-9 formed suprastructures as sheets; these freestanding JNP sheets have low curvature and their area could be as large as hundreds of micrometers square (Figure 4c). Both single- and double-layered structures were observed. A detailed examination could clearly reveal the hexagonally and closely packed JNP-9 with high orientation (Figure 4e,f). The influence of directional angle $\alpha$ and aspect ratio $\gamma$ of JNPs on their assembly is summarized in Figure 5 and in Table 1.

Comparison with Computer Simulation Results. It would be instructive at this point to confront the current experimental findings with the simulation results. First, we compare our results with the computer simulations of the selfassembly of spherical Janus colloids and next with the selfassembly of dumbbell Janus particles. In a previous work, Sciortino et al. ${ }^{31}$ performed numerical simulations on the assembly of spherical Janus colloids consisting of an attractive and a repulsive patch as a function of concentration and temperature. The simulations were carried on Janus particles with a fixed ratio between the area of the attractive patch to the entire area of the sphere, namely, a hemispherical patch $\chi=0.5$. 
Table 1. Summary of the Experimentally Observed Assembled Suprastructures' Function of JNPs' Aspect Ratios and Directional Angles

\begin{tabular}{|c|c|c|c|}
\hline$\gamma$ & $\begin{array}{c}\alpha \\
(\mathrm{deg})\end{array}$ & type of structure & size of the structure \\
\hline \multirow[t]{5}{*}{$1.2-1.3$} & 66.2 & spherical micelles & $\begin{array}{l}1.3 \pm 0.5 \mu \mathrm{m}(\text { in } \\
\text { diameter })\end{array}$ \\
\hline & 26.6 & $\begin{array}{l}\text { monowalled capsules/ } \\
\text { vesicles }\end{array}$ & $\begin{array}{l}3.7 \pm 1.9 \mu \mathrm{m}(\text { in } \\
\text { diameter })\end{array}$ \\
\hline & 12.6 & $\begin{array}{l}\text { monowalled capsules/ } \\
\text { vesicles or curved } \\
\text { monolayers }\end{array}$ & $\begin{array}{l}6.4 \pm 1.1 \mu \mathrm{m}(\text { in } \\
\text { diameter })\end{array}$ \\
\hline & 6.2 & planar monolayer islands & $0.5 \pm 0.4 \mu \mathrm{m}^{2}$ (area) \\
\hline & 0.2 & planar bilayer/monolayers & $1-80 \mu \mathrm{m}^{2}$ (area) \\
\hline \multirow[t]{2}{*}{$1.3-1.4$} & 33.7 & $\begin{array}{l}\text { monowalled capsules/ } \\
\text { vesicles with inversely } \\
\text { oriented JNPs }\end{array}$ & $\begin{array}{l}21.4 \pm 5.9 \mu \mathrm{m} \text { (in } \\
\text { diameter of collapsed } \\
\text { capsules) }\end{array}$ \\
\hline & 40.9 & random aggregates & \\
\hline \multirow[t]{2}{*}{$1.4-1.6$} & 5 & random aggregates & \\
\hline & -38.6 & $\begin{array}{l}\text { capsules with randomly } \\
\text { oriented JNPs }\end{array}$ & $\begin{array}{l}1.9 \pm 1.2 \mu \mathrm{m}(\text { in } \\
\text { diameter })\end{array}$ \\
\hline$>1.6$ & -37.4 & random aggregates & \\
\hline
\end{tabular}

For $\chi=0.5$ the most frequent clusters were the single-layer micelles, with an average of 10 Janus particles oriented such that the attractive patch is buried inside the structure. Bilayer vesicles containing an average of 40 particles were also predicted. The orientation of the particles in the micelles is similar to our observations; however, we only observed clusters with single-layer walls. When the ratio between the area of the attractive patch to the entire area of the sphere decreased to $\chi=$ 0.4 , micelles still formed but a lamellar phase became dominant especially at higher colloid concentrations. ${ }^{37}$ For $\chi=0.3$ a new phase, namely, long wires/worms, appeared competing with the formation of micelles, and for $\chi=0.2$ and 0.1 micelles mostly single particles were formed. These can be compared with the experimental findings of Granick et al. ${ }^{38,39}$ While a one-to-one comparison is difficult, we highlight some important differences in the assembly behavior of the snowman JNPs to the spherical patchy Janus colloids. In our case, with the decrease in the relative size of the attractive lobe we observe that structures with higher curvature are preferred, as seen in Figure 5. In the case of the spherical Janus colloids the formation of spherical micelles is preferred for the case of $\chi=0.5$. The structural diversity appears with $\chi<0.5$ but without a clear dependence on the curvature of the produced suprastructures. In our case the formation of the higher curvature structures, micelles, appears to be preferred when the attractive lobe is smallest. From these observations it becomes obvious that the two-lobe JNPs differentiate themselves from the spherical Janus colloids through a strong directionality given by their geometry, while in the case of spherical Janus colloids the assembly behavior is purely defined by the attraction-repulsion balance.

Our experimental findings that are summarized in Figure 5 and Table 1 are better reflected in a very recent numerical Monte Carlo simulations by O'Toole et al. ${ }^{40,41}$ The authors investigated the assembly of dumbbell-type JNPs consisting of one repulsive hard sphere (diameter $\sigma_{\mathrm{h}}$ ) and one attractive square well (diameter $\sigma_{\mathrm{s}}$ ) sphere. They parametrized the relative dimensions between the lobes by a size ratio parameter $\theta$ (original notation used was $\alpha$ ), defined as $\sigma_{\mathrm{s}} / \sigma_{\mathrm{h}}$ for $\theta<1$ and $\left(2 \sigma_{\mathrm{s}}-\sigma_{\mathrm{h}}\right) / \sigma_{\mathrm{s}}$ for $\theta>1$, with the limiting case $\theta=1$ equal to the Janus lobe sizes. Planar two-dimensional bilayers were predicted for $\theta=1$. Upon decreasing the lobe size $\sigma_{\mathrm{s}}$ such that $\theta=2 / 3$, micelles and platelets begin forming. For $\theta<1 / 2$, highly ordered polyhedrons and spherical micelles are formed due to the packing restrictions as $\sigma_{\mathrm{s}}$ decreases. Oppositely, for $\theta$ $>1$, the formation of reversed micelles coexists with other structures, but no details exist on the orientation of particles. ${ }^{41}$ Compared to our findings, following along the $x$-axis of the graph in Figure 5, notable differences with the above results are observed. In our case, we observe, with the relative increase of the attractive lobe, the formation of spherical structures with lower curvatures, from micelles to vesicles and from vesicles to very large capsules with ordered JNPs. In the computer simulation mentioned above, instead of spherical structures, progressively larger platelets were observed. Another evident difference is that as for $\theta>1$, we observed capsules with walls consisting of randomly oriented JNPs rather than the predicted inverse micelles. Micelles formed by snowman-type JNPs were reported in an experimental work by Kraft et al., ${ }^{22}$ but they did not observe larger monowalled vesicles and capsules. In light of limited data on experimental works on the assembly of snowman or dumbbell JNPs, we believe this to be a prime experimental report observing such large- and low-curvature structures consisting of highly ordered JNPs assembled in a monophasic solvent. It should be noted that the landscape of suprastructures formed by the assembly of Janus colloids in 2D appears to be as rich, ${ }^{42-44}$ but because these are bidimensional suprastructures are very different from our 3D assemblies, an extensive discussion is not the subject of this report.

Influence of the JNPs Size on JNP Self-Assembly. Size is an important parameter for nanoparticles that could also influence their self-assembly behaviors. In order to examine the influence of JNP size on their self-assembly properties, we synthesized JNP-10. As compared to JNP-9, JNP-10 has a very similar geometry both in directionality $\left(\alpha=6.2^{\circ}\right)$ and aspect ratio $(\gamma=1.22)$; however, JNP-10 is only about half the size of JNP-9. Therefore, similarities in the formed self-assembled structures can be found between JNP-9 and JNP-10. For example, JNP-10 tends to assemble into planar suprastructures consisting of highly oriented JNPs (Figure $4 \mathrm{~g}$ ). However, the self-assembly of JNP-10 has its own characteristics; namely, the planar structures formed by JNP-10 are mostly single layered and appear significantly less compact. This phenomenon ought to be the consequence of the smaller dimensions of JNP-10 as compared to those of JNP-9. On the one hand, the reduced PtBA interacting lobe size implies a decreased contacting area between JNPs and, therefore, a reduced hydrophobic attraction force, which is essentially responsible for holding together the assembled suprastructures. On the other hand, the reduced size also means a higher mobility of JNPs; thus the resulting selfassembled structures are more prone to be ripped apart by shearing forces or capillary forces during the drying process. The particle islands assembled by JNP-10 (Figure 4e) instead of a very compact film formed by JNP-9 (Figure 4d) is the aftermath of the capsule's inability to maintain its full integrity during sample processing, as a consequence of reduced dimensions.

Coassembly of JNPs with Different Geometries. Thus far, we have shown the different assembly properties of JNPs because of their different geometries. Coassembly of different colloidal particles is an important way to make complex hierarchical supracolloidal structures. ${ }^{45}$ Additionally, coassembly may lead to properties that individual colloidal particles cannot achieve. It is fundamentally interesting to study such coassembly properties and the suprastructures resulting from 

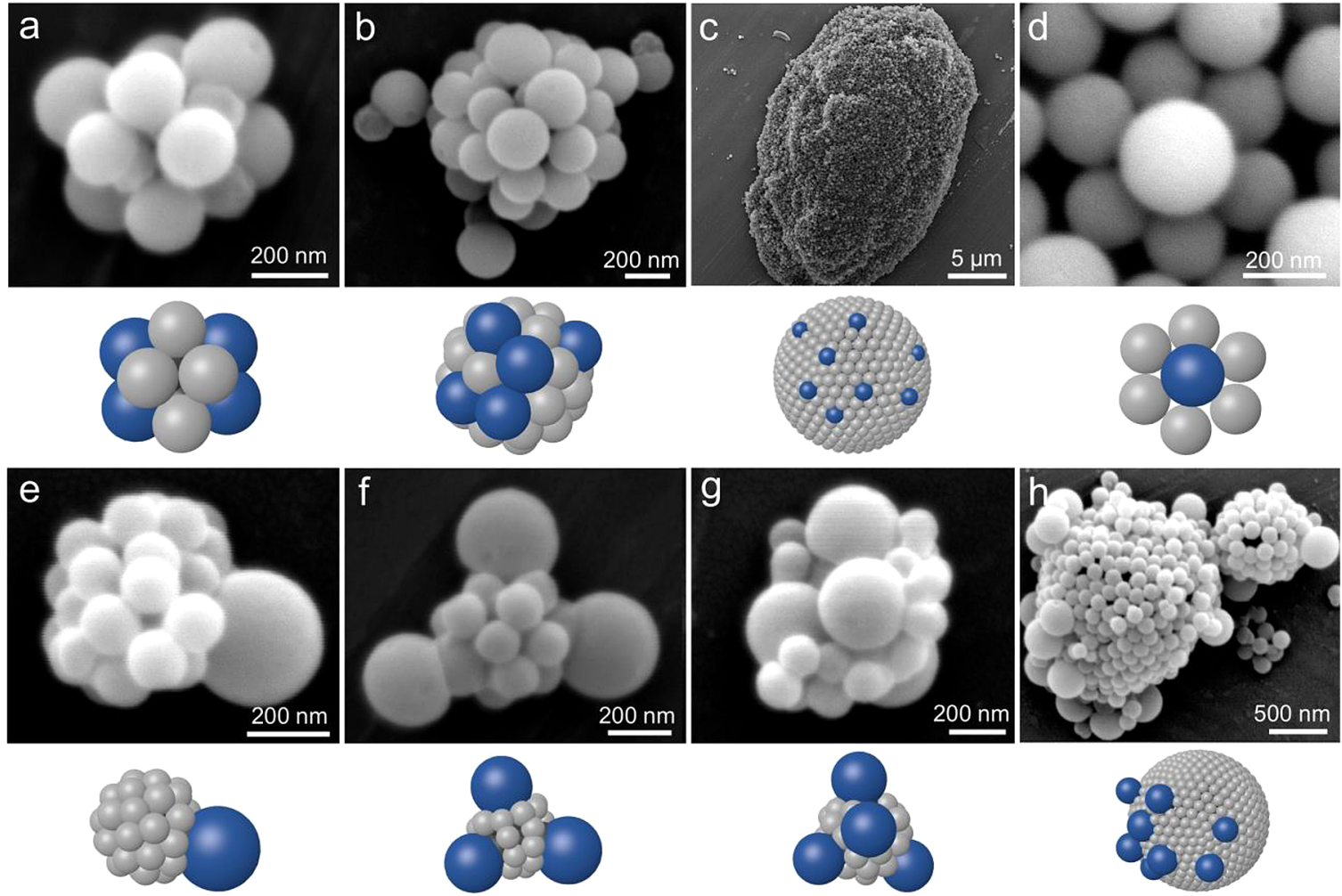

Figure 6. Suprastructures obtained from the coassembly of JNPs. (a-c) Micelles, minicapsules, and giant capsules from the coassembly of JNP-1 and JNP-5 (blue). (d) Magnified image of (c). (e-h) Suprastructures coassembled by JNP-5 (blue) and JNP-10.

the JNPs having different geometries. Previously, colloidal particles of different sizes $^{46}$ and directionalities ${ }^{47}$ have been shown to form self-assemblies; however, the coassembly of different nanoparticles with anisotropic geometries so as to form complex structures has not been reported yet. Here, we show the coassembly of JNPs. In particular, we are especially interested in the coassembly of JNPs, which exhibit differences in directionality $\alpha$, aspect ratio $\gamma$, and overall size. For this, we studied the coassembly of JNP-1 $\left(\alpha=66.2^{\circ}\right.$ and $\left.\gamma=1.21\right)$ with JNP-5 ( $\alpha=40.9^{\circ}$ and $\left.\gamma=1.26\right)$, which differ especially in the phase separation and overall size but have similar directionality. From Figure 6 we can see that for the mass ratio of 3:5 (JNP5:JNP-1), JNP-5 and JNP-1 can indeed coassemble to form a surprisingly large variety of ordered suprastructures, including micelles, minicapsules, and vesicles, Figure $6 a-c$. Additionally, because of the obvious size differences, one can easily distinguish between JNP-1 and JNP-5 in the coassembly structures, which are both highly oriented. A detailed examination indicates that, in contrast to the structures formed by JNP-5 alone, JNP-5 adopts a highly oriented manner to fit in the coassembled structures by pointing the PtBA lobe inward and the PTPM lobe toward the aqueous solution (Figure 6ad). In addition we studied the coassembly of JNP-5 ( $\alpha=40.9^{\circ}$ and $\gamma=1.26)$ with JNP-10 $\left(\alpha=6.2^{\circ}\right.$ and $\left.\gamma=1.21\right)$; mass ratio, JNP-5:JNP-10 $=3: 5$. From Figure $6 \mathrm{e}-\mathrm{h}$ we can see that even though JNP-5 and JNP-10 are very different in their dimensions, regular coassembled structures could be obtained such as micelles, minicapsules, and vesicles. In these coassembled structures, JNP-5 JNPs are highly oriented, and the smaller JNP-10 interacts with JNP-5 through their PtBA lobe. While only planar self-assembled monolayers could be obtained from JNP-10 alone, the large size of JNP-5 and its larger $\alpha$ lead to an increased curvature of the suprastructures.
With the increasing proportion of JNP-5 to JNP-10, the curvature of the suprastructure formed by coassembly also increases. The variation in the proportion of JNP-5 to JNP-10 also confirms that a large variety of suprastructures could possibly be obtained.

\section{CONCLUSIONS}

The effect of geometries in colloidal assembly is of prime interest for the fabrication of supracolloidal aggregates with specific structures and functionalities. In the present study, we have synthesized 10 types of JNPs with identical chemical compositions but of different geometries. We highlighted the importance of some key geometric parameters such as JNPs' directionality and aspect ratio that determine the types of colloidal assembly that can be obtained. Responsible for particle assembly and suprastructure formation is the attractive hydrophobic interaction between the PtBA Janus lobes and the packing possibilities around it. When the PTMP nonattractive lobe is larger than the PtBA lobe, the JNPs have a high directionality ( $\alpha$ is large) due to restricted rotation possibility and bonding points around the latter lobe; thus these JNPs are prone to assemble into well-defined highly oriented suprastructures. The decrease in $\alpha$ values leads to the formation of free-standing single- or double-layered JNP sheets having a low curvature. Below a critical value, $\alpha<0$, the JNPs are still capable of self-assembling in capsules, but the degree of JNP rotational freedom increases, giving rise to disorder. We have shown that the aspect ratio $\gamma$ of JNPs, which is controlled by the degree of phase separation, also plays an important role. It turns out that a lesser aspect ratio ( small $\gamma$ ) is more favorable for suprastructures formation. The coassembly of JNPs with different geometries represents an interesting example of the coassembly between colloidal particles with anisotropic geo- 
metries with high orientation. We found out coassembly could produce a much larger diversity of suprastructures as compared to those obtained from individual JNPs.

\section{METHODS}

Synthesis of PtBA-PTPM JNPs was done via seeded emulsion polymerization and phase separation from two batches of surfactantfree PtBA seed nanoparticles of $139 \pm 6 \mathrm{~nm}$ and $83 \pm 4 \mathrm{~nm}$ in dried state, as described in the Supporting Information (SI). The larger diameter PtBA seed nanoparticle were used to prepare the JNP-1-9 series, and the smaller diameter seed nanoparticles were used for the synthesis of JNP-10. By adjusting the volume of the 3-(triethoxysilyl)propyl methacrylate monomer to $1 \mathrm{~g}$ of PtBA seed nanoparticles, two series of JNPs with varying PTPM lobe sizes were obtained. In the JNP-1-4 series, the volume of TPM used for $1 \mathrm{~g}$ of PtBA seed NPs was $2 \mathrm{~mL}$ for JNP-1 (PTPM lobe diameter $209 \pm 11 \mathrm{~nm}$ ), $1.5 \mathrm{~mL}$ for JNP-2 (PTPM lobe diameter $180 \pm 6 \mathrm{~nm}$ ), $1 \mathrm{~mL}$ for JNP-3 (PTPM lobe diameter $162 \pm 6 \mathrm{~nm}$ ), and $0.5 \mathrm{~mL}$ for JNP-4 (PTPM lobe diameter $122 \pm 8 \mathrm{~nm}$ ); for the JNP-5-8 series, the volume of TPM for $1 \mathrm{~g}$ of seed nanoparticle used was $2.5 \mathrm{~mL}$ for JNP-5 (PTPM lobe diameter $238 \pm 6 \mathrm{~nm}$ ), $2 \mathrm{~mL}$ for JNP-6 (PTPM lobe diameter $209 \pm 5$ $\mathrm{nm}$ ), $1 \mathrm{~mL}$ for JNP-7 (PTPM lobe diameter $161 \pm 4 \mathrm{~nm}$ ), and $0.5 \mathrm{~mL}$ for JNP-8 (PTPM lobe diameter $116 \pm 4 \mathrm{~nm}$ ). The increase in phase separation between the JNP lobes was achieved by an increase in the polymerization time of the seed nanoparticles, $2 \mathrm{~h}$ for JNP-9 (length $186 \pm 10 \mathrm{~nm}$ ), $8 \mathrm{~h}$ for JNP-1 (length $255 \pm 10 \mathrm{~nm}$ ), and $24 \mathrm{~h}$ for JNP5 (length $317 \pm 12 \mathrm{~nm}$ ). Particle sizes were determined by SEM; see more in the SI. The self-assembly procedure of the obtained PtBAPTPM JNPs was carried out in water with $10 \mathrm{vol} \%$ ethanol, at a particle concentration of $10.0 \mathrm{mg} / \mathrm{mL}$, under shear produced by shaking or sonication at room temperature as described in the SI. The colloidal suprastructures obtained from JNPs with different geometries and sizes were characterized in the dry state by SEM and in situ by optical fluorescence microscopy; see more in the SI. The capsules and vesicles were characterized in situ in the assembly solution by an inverted fluorescence microscope (IX51, Olympus, Tokyo, Japan), equipped with a $100 \mathrm{~W}$ mercury discharge lamp (USH-103OL Ushio, Tokyo Japan), a 460-495 nm excitation filter, and a CPLN-PH 60X microscopy objective. The hydrophilic rhodamine $\mathrm{B}$ dye was used as fluorophore.

\section{ASSOCIATED CONTENT}

\section{S Supporting Information}

The Supporting Information is available free of charge on the ACS Publications website at DOI: 10.1021/acsnano.8b00960.

Description of seed PtBA NPs and PtBA-PTPM JNP synthesis procedures; crystallization method for determining the swelling degree of PtBA NPs in ethanol; procedure for self-assembly of JNPs; characterization methods for JNPs and their assemblies; SEM images of broken capsules, fluorescence microscopy images of the self-assembled capsules; wide-field SEM image of the self-assembled JNP capsules; SEM image of the swelled crystal of PtBA NPs as assembled in ethanol showing shrinkage after drying (PDF)

\section{AUTHOR INFORMATION}

\section{Corresponding Author}

*E-mail: andrei.honciuc@zhaw.ch.

\section{ORCID $\odot$}

Chengjun Kang: 0000-0003-0208-2954

Andrei Honciuc: 0000-0003-2160-2484

\section{Notes}

The authors declare no competing financial interest.

\section{ACKNOWLEDGMENTS}

We gratefully acknowledge the financial funding from Metrohm Foundation (Herisau, Switzerland).

\section{REFERENCES}

(1) Kim, S.-H.; Lee, S. Y.; Yang, S.-M.; Yi, G.-R. Self-Assembled Colloidal Structures for Photonics. NPG Asia Mater. 2011, 3, 25-33.

(2) Yethiraj, A.; Thijssen, J. H. J.; Wouterse, A.; van Blaaderen, A. Large-Area Electric-Field-Induced Colloidal Single Crystals for Photonic Applications. Adv. Mater. 2004, 16, 596-600.

(3) Anker, J. N.; Hall, W. P.; Lyandres, O.; Shah, N. C.; Zhao, J.; Van Duyne, R. P. Biosensing with Plasmonic Nanosensors. Nat. Mater. 2008, 7, 442-453.

(4) Fenzl, C.; Hirsch, T.; Wolfbeis, O. S. Photonic Crystals for Chemical Sensing and Biosensing. Angew. Chem., Int. Ed. 2014, 53, 3318-3335.

(5) Ortel, E.; Sokolov, S.; Zielke, C.; Lauermann, I.; Selve, S.; Weh, K.; Paul, B.; Polte, J.; Kraehnert, R. Supported Mesoporous and Hierarchical Porous $\mathrm{Pd} / \mathrm{TiO} 2$ Catalytic Coatings with Controlled Particle Size and Pore Structure. Chem. Mater. 2012, 24, 3828-3838.

(6) Sacanna, S.; Irvine, W. T. M.; Chaikin, P. M.; Pine, D. J. Lock and Key Colloids. Nature 2010, 464, 575-578.

(7) Damasceno, P. F.; Engel, M.; Glotzer, S. C. Predictive SelfAssembly of Polyhedra into Complex Structures. Science 2012, 337, 453-457.

(8) Nguyen, T. D.; Jankowski, E.; Glotzer, S. C. Self-Assembly and Reconfigurability of Shape-Shifting Particles. ACS Nano 2011, 5, 8892-8903.

(9) Forster, J. D.; Park, J.-G.; Mittal, M.; Noh, H.; Schreck, C. F.; O'Hern, C. S.; Cao, H.; Furst, E. M.; Dufresne, E. R. Assembly of Optical-Scale Dumbbells into Dense Photonic Crystals. ACS Nano 2011, 5, 6695-6700.

(10) Zhang, Y.; Lu, F.; van der Lelie, D.; Gang, O. Continuous Phase Transformation in Nanocube Assemblies. Phys. Rev. Lett. 2011, 107, 135701.

(11) Glotzer, S. C.; Solomon, M. J. Anisotropy of Building Blocks and Their Assembly into Complex Structures. Nat. Mater. 2007, 6, $557-562$.

(12) Gunton, J. D.; Shiryayev, A.; Pagan, D. L. Protein Condensation: Kinetic Pathways to Crystallization and Disease; Cambridge University Press: New York, 2007.

(13) Liu, Y.; Li, W.; Perez, T.; Gunton, J. D.; Brett, G. Self Assembly of Janus Ellipsoids. Langmuir 2012, 28, 3-9.

(14) Li, W.; Gunton, J. D. Self-Assembly of Janus Ellipsoids II: Janus Prolate Spheroids. Langmuir 2013, 29, 8517-8523.

(15) Sun, Y.; Xia, Y. Shape-Controlled Synthesis of Gold and Silver Nanoparticles. Science 2002, 298, 2176-2179.

(16) Greyson, E. C.; Barton, J. E.; Odom, T. W. Tetrahedral Zinc Blende Tin Sulfide Nano- and Microcrystals. Small 2006, 2, 368-371.

(17) Lee, S.-M.; Jun, Y.; Cho, S.-N.; Cheon, J. Single-Crystalline StarShaped Nanocrystals and Their Evolution: Programming the Geometry of Nano-Building Blocks. J. Am. Chem. Soc. 2002, 124, 11244-11245.

(18) Kim, S.-H.; Abbaspourrad, A.; Weitz, D. A. Amphiphilic Crescent-Moon-Shaped Microparticles Formed by Selective Adsorption of Colloids. J. Am. Chem. Soc. 2011, 133, 5516-5524.

(19) Fan, J.-B.; Song, Y.; Liu, H.; Lu, Z.; Zhang, F.; Liu, H.; Meng, J.; Gu, L.; Wang, S.; Jiang, L. A General Strategy to Synthesize Chemically and Topologically Anisotropic Janus Particles. Sci. Adv. 2017, 3, 1603203.

(20) Manoharan, V. N. Colloidal Matter: Packing, Geometry, and Entropy. Science 2015, 349, 1253751.

(21) Hu, H.; Ji, F.; Xu, Y.; Yu, J.; Liu, Q.; Chen, L.; Chen, Q.; Wen, P.; Lifshitz, Y.; Wang, Y.; Zhang, Q.; Lee, S. Reversible and Precise Self-Assembly of Janus Metal-Organosilica Nanoparticles through a Linker-Free Approach. ACS Nano 2016, 10, 7323-7330.

(22) Kraft, D. J.; Ni, R.; Smallenburg, F.; Hermes, M.; Yoon, K.; Weitz, D. A.; van Blaaderen, A.; Groenewold, J.; Dijkstra, M.; Kegel, 
W. K. Surface Roughness Directed Self-Assembly of Patchy Particles into Colloidal Micelles. Proc. Natl. Acad. Sci. U. S. A. 2012, 109, 10787-10792.

(23) Evers, C. H. J.; Luiken, J. A.; Bolhuis, P. G.; Kegel, W. K. SelfAssembly of Microcapsules via Colloidal Bond Hybridization and Anisotropy. Nature 2016, 534, 364-368.

(24) Ducrot, É.; He, M.; Yi, G.-R.; Pine, D. J. Colloidal Alloys with Preassembled Clusters and Spheres. Nat. Mater. 2017, 16, 652-657.

(25) Sacanna, S.; Korpics, M.; Rodriguez, K.; Colón-Meléndez, L.; Kim, S.-H.; Pine, D. J.; Yi, G.-R. Shaping Colloids for Self-Assembly. Nat. Commun. 2013, 4, 1688-1694.

(26) Walther, A.; Müller, A. H. E. Janus Particles: Synthesis, SelfAssembly, Physical Properties, and Applications. Chem. Rev. 2013, 113, 5194-5261.

(27) Vogel, N.; Retsch, M.; Fustin, C.-A.; del Campo, A.; Jonas, U. Advances in Colloidal Assembly: The Design of Structure and Hierarchy in Two and Three Dimensions. Chem. Rev. 2015, 115, 6265-6311.

(28) Tang, C.; Zhang, C.; Liu, J.; Qu, X.; Li, J.; Yang, Z. Large Scale Synthesis of Janus Submicrometer Sized Colloids by Seeded Emulsion Polymerization. Macromolecules 2010, 43, 5114-5120.

(29) Pham, B. T.; Such, C.; Hawkett, B. Synthesis of Polymeric Janus Nanoparticles and Their Application in Surfactant-Free Emulsion Polymerizations. Polym. Chem. 2015, 6, 426-435.

(30) Avvisati, G.; Vissers, T.; Dijkstra, M. Self-Assembly of Patchy Colloidal Dumbbells. J. Chem. Phys. 2015, 142, 084905.

(31) Sciortino, F.; Giacometti, A.; Pastore, G. Phase Diagram of Janus Particles. Phys. Rev. Lett. 2009, 103, 237801.

(32) Vissers, T.; Preisler, Z.; Smallenburg, F.; Dijkstra, M.; Sciortino, F. Predicting Crystals of Janus Colloids. J. Chem. Phys. 2013, 138, 164505.

(33) Chen, W.-H.; Tu, F.; Bradley, L. C.; Lee, D. Shape-Tunable Synthesis of Sub-Micrometer Lens-Shaped Particles via Seeded Emulsion Polymerization. Chem. Mater. 2017, 29, 2685-2688.

(34) Wu, D.; Chew, J. W.; Honciuc, A. Polarity Reversal in Homologous Series of Surfactant-Free Janus Nanoparticles: Toward the Next Generation of Amphiphiles. Langmuir 2016, 32, 6376-6386.

(35) Wu, D.; Honciuc, A. Design of Janus Nanoparticles with PHTriggered Switchable Amphiphilicity for Interfacial Applications. ACS Appl. Nano Mater. 2018, 34, 1225-1233.

(36) Israelachvili, J. N. Intermolecular and Surface Forces, 3rd ed.; Academic Press: San Diego, 2011.

(37) Munaò, G.; Preisler, Z.; Vissers, T.; Smallenburg, F.; Sciortino, F. Cluster Formation in One-Patch Colloids: Low Coverage Results. Soft Matter 2013, 9, 2652-2661.

(38) Hong, L.; Cacciuto, A.; Luijten, E.; Granick, S. Clusters of Amphiphilic Colloidal Spheres. Langmuir 2008, 24, 621-625.

(39) Chen, Q.; Whitmer, J. K.; Jiang, S.; Bae, S. C.; Luijten, E.; Granick, S. Supracolloidal Reaction Kinetics of Janus Spheres. Science 2011, 331, 199-202.

(40) O’Toole, P.; Munaò, G.; Giacometti, A.; Hudson, T. S. SelfAssembly Behaviour of Hetero-Nuclear Janus Dumbbells. Soft Matter 2017, 13, 7141-7153.

(41) O’Toole, P.; Giacometti, A.; Hudson, T. Phase Diagram of Heteronuclear Janus Dumbbells. Soft Matter 2017, 13, 803-813.

(42) Iwashita, Y.; Kimura, Y. Stable Cluster Phase of Janus Particles in Two Dimensions. Soft Matter 2013, 9, 10694-10698.

(43) Sabapathy, M.; Remya, A. M.; Mani, E. Self-Assembly of Inverse Patchy Colloids with Tunable Patch Coverage. Phys. Chem. Chem. Phys. 2017, 19, 13122-13132.

(44) Shin, H.; Schweizer, K. S. Theory of Two-Dimensional SelfAssembly of Janus Colloids: Crystallization and Orientational Ordering. Soft Matter 2013, 10, 262-274.

(45) Gröschel, A. H.; Walther, A.; Löbling, T. I.; Schacher, F. H.; Schmalz, H.; Müller, A. H. E. Guided Hierarchical Co-Assembly of Soft Patchy Nanoparticles. Nature 2013, 503, 247-251.

(46) Cho, Y.-S.; Yi, G.-R.; Lim, J.-M.; Kim, S.-H.; Manoharan, V. N.; Pine, D. J.; Yang, S.-M. Self-Organization of Bidisperse Colloids in Water Droplets. J. Am. Chem. Soc. 2005, 127, 15968-15975.
(47) Wang, Y.; Wang, Y.; Breed, D. R.; Manoharan, V. N.; Feng, L.; Hollingsworth, A. D.; Weck, M.; Pine, D. J. Colloids with Valence and Specific Directional Bonding. Nature 2012, 491, 51-55. 